\title{
Spontaneous ventilation thoracoscopic thymectomy: attractive or exceptionable?
}

\author{
Eugenio Pompeo ${ }^{1}$, Ahmed G. Elkhouly ${ }^{2}$ \\ ${ }^{1}$ Department of Thoracic Surgery, Policlinico Tor Vergata University, Rome, Italy; ${ }^{2}$ Department of Cardiothoracic Surgery, Faculty of Medicine, \\ Tanta University, Tanta, Egypt \\ Correspondence to: Eugenio Pompeo, MD, FETCS. Department of Thoracic Surgery, Policlinico Tor Vergata University, V.le Oxford, 81, 00133 \\ Rome, Italy. Email: pompeo@med.uniroma2.it. \\ Comment on: Jiang L, Depypere L, Rocco G, et al. Spontaneous ventilation thoracoscopic thymectomy without muscle relaxant for myasthenia gravis: \\ Comparison with "standard" thoracoscopic thymectomy. J Thorac Cardiovasc Surg 2018;155:1882-9.e3.
}

Submitted Aug 28, 2018. Accepted for publication Sep 12, 2018.

doi: $10.21037 /$ jtd.2018.09.75

View this article at: http://dx.doi.org/10.21037/jtd.2018.09.75

Thymectomy has proven beneficial in myasthenia gravis (MG) confirming its complimentary role to pharmacological therapy including corticosteroids in patients with generalized disease or in those with thymomatous MG (1).

The optimal surgical approach for thymectomy is still widely debated due to the existence of different open transcervical, trans-sternal and combined approaches, as well as of several thoracoscopic alternatives, which comprise today right-sided, left sided, subxiphoid, transcervical and combined approaches, some of which can be also accomplished by robot-assisted surgery (2). Moreover, when taking into account the surgical management of MG patients, the optimal anesthesia regimen poses particular demand. This is because by standard general anesthesia protocols with double-lumen tube intubation and mechanical ventilation, postoperative respiratory complications including the development of myasthenic crisis (MC) in up to $27 \%$ of operated patients have been reported (3).

In order to limit these risks, use of short-acting muscle relaxants with train of four monitoring (4) and even avoidance of muscle relaxants (5) have been suggested in MG patients undergoing thymectomy. In addition, anecdotal reports have suggested potential advantages in thymectomy carried out by spontaneous ventilation anesthesia protocols $(1,6,7)$.

We have read with great interest the retrospective analysis on thoracoscopic thymectomy under spontaneous ventilation published by Jiang and coworkers (3) in the
April issue of the Fournal of Thoracic and Cardiovascular Surgery.

In their interesting study, the authors have compared, with the aid of a propensity score matching analysis, two cohorts of patients undergoing either spontaneousventilation video-assisted thoracic thymectomy (SV-VATT) or intubated VATT (IVATT). The study results have shown comparable perioperative outcomes with lower incidence of $\mathrm{MC}$ and of postoperative mechanical ventilation, lower pain scores, as well as a shorter hospital stay in the SV-VATT group. The authors' conclusions were that SV-VATT is feasible and may be beneficial by offering lesser risks in the early postoperative period for MG patients.

With this recent publication the surgical team from Guangzhou, which has demonstrated a consolidated expertise in non-intubated thoracic surgery procedures, reported very interesting data points, based on the largest series published so far on thymectomy performed under spontaneous ventilation. In fact, the first report on awake thoracoscopic thymectomy goes back to 2004 when Tsunezuka et al. (8) reported on extended thymectomy, which was performed under sole thoracic epidural anesthesia by a median sternotomy. Subsequently, Al-Abdullatief and coworkers (6) reported on 25 patients who were operated on through a left-sided thoracoscopic approach whereas in 2008, Matsumoto et al. (9) developed a subxiphoid approach with sternal lifting, for an awake thymectomy. More recently, we have reported on a small series of patients with thymomatous MG who had undergone a minimalist right- 
sided thoracoscopic thymectomy (7).

From a purely physiological point of view, non-intubated anesthesia with maintenance of spontaneous ventilation, might be considered ideal. Nonetheless, by this anesthesia protocol concerns may be raised as to the maintenance of rhythmic diaphragmatic movements, can disturb somewhat surgical maneuvering. Moreover, there exists also a risk of a delayed conversion to tracheal intubation and emergency sternotomy if prompt management of a major vascular injury is required.

In fact, in initial series dealing with non-intubated thymectomy performed under spontaneous ventilation, the adoption of thoracic epidural anesthesia in fully awake patients added another limitation related to the need of maintaining a negative pressure environment at least in one of the pleural cavities. This to assure a satisfactory ventilation, which might have been jeopardized in case of inadvertent opening of both mediastinal pleurae. On the other hand, the evolutionary anesthesia protocols, which have been preferred in more recent reports by us (7) as well as by Dr. Jiang and coworkers (3) entail the additional use of a laryngeal mask with sedation, which can allow mechanical assistance of ventilation whenever needed in order to avoid intra-operative oxygenation problems. This aspect should not be underestimated, particularly when removal of the thymus gland is planned to be associated with that of extrathymic fatty tissue within the anterior mediastinum, which has been shown to contain ectopic thymic tissue in $21 \%$ up to $63 \%$ of instances (10). Removal of this tissue is recommended to accomplish a formal extended thymectomy (11). This is done according to the believe that the more complete is the removal of thymic tissue, the better is the chance of a postoperative improvement in MG symptoms.

A further unresolved debate regards the side from which the thymus should be approached thoracoscopically. In their series, Dr. Jiang et al (3) preferred a right-sided surgical approach, which is thought to allow easier dissection maneuvers to right-handed surgeons and to easily identify the confluence of the left innominate vein with superior vena cava, which represents a useful landmark to facilitate identification and division of the thymic veins when thymectomy is being accomplished (12). On the contrary, defenders of a left-sided approach indicate that advantages of this approach include the finding that the left lower thymic horn is usually larger than the contralateral one and that the aorto-pulmonary window, which can also contain ectopic thymic tissue, can be more directly reached (13). To complicate this even more in regards to the scenario, thoracoscopic subxiphoid approaches (14) have also been proposed to gain an optimal exposure of both lateral aspects of the anterior-superior mediastinum.

We believe that irrespective from the chosen side to approach the thymus, the main challenge of thoracoscopic approaches is that of achieving an optimal exposure of the lower cervical area and dissect free the superior thymic horns, due to the narrow space existing at this level when operating in a closed chest. This feature has led to advocate additional technical tricks including creation of an adjuvant pneumomediastinum (15) and of accessory cervical incisions with sternal lifting to facilitate surgical dissection of this area (4).

As a result of this multifaceted scenario, superiority of a single surgical approach is far to be identified, and the choice is usually based on individual preferences and aptitudes of each of the thoracic surgeons.

When looking at the indications to thymectomy, it is worth noting that in the series from Jiang et al. (3), $40 \%$ and $48 \%$ of operated patients in the SV-VATT and IVATT groups, respectively, had pure ocular MG. If we exclude patients with an ocular MG associated with thymoma in whom the rationale for thymectomy is mainly oncological and thus widely accepted, the usefulness of thymectomy in patients with non-thymomatous ocular MG, is controversial. Nonetheless, in our experience we have found no difference in improvement rates amongst patients with generalized or ocular MG in a series of patients who had undergone a thoracoscopic extended thymectomy (4).

Regarding the analysis of early results, in the study from Jiang et al. (3), there was no mortality in either group whereas morbidity, which included the occurrence of MC was lower in the SV-VATT group. This result is broadly corroborated by other literature data sets, which showed no operative mortality following thoracoscopic thymectomy $(1,4,16)$.

MG scores at patient's discharge were similar between the study groups (3) demonstrating that the type of surgical approach did not affect symptoms behavior after surgery. Hence, it will be interesting to see the long-term results of these cohorts, in order to verify equivalence or superiority of one of the surgical approaches in terms of MG symptoms' remission and improvement. In addition, the potential operative advantages which were hypothesized for SV-VATT should prove to be associated at least by an equivalence with other alternative open and thoracoscopic approaches in terms of oncological results including longterm survival in patients with thymoma. 
In conclusions, Dr. Jiang and coworkers (3) are to be congratulated for their continuing excellent investigation on the potential advantages and indications for thoracoscopic procedures which were performed under spontaneous ventilation without intubation.

Time and greater experience with the SV-VATT, will eventually clarify the ongoing debate. So far, we would argue that probably, for quite many thoracic surgeons, this surgical procedure will prove to be both attractive and exceptionable at the same time and this might be considered a highly satisfactory result already.

\section{Acknowledgements}

None.

\section{Footnote}

Conflicts of Interest: The authors have no conflicts of interest to declare.

\section{References}

1. Wolfe GI, Kaminski HJ, Aban IB, et al. Randomized Trial of Thymectomy in Myasthenia Gravis. N Engl J Med 2016;375:511-22.

2. Sonett JR, Magee MJ, Gorenstein L. Thymectomy and myasthenia gravis: A history of surgical passion and scientific excellence. J Thorac Cardiovasc Surg 2017;154:306-9.

3. Jiang L, Depypere L, Rocco G, et al. Spontaneous ventilation thoracoscopic thymectomy without muscle relaxant for myasthenia gravis: Comparison with "standard" thoracoscopic thymectomy. J Thorac Cardiovasc Surg 2018;155:1882-9.e3.

4. Pompeo E, Tacconi F, Massa R, et al. Long-term outcome of thoracoscopic extended thymectomy for nonthymomatous myasthenia gravis. Eur J Cardiothorac Surg 2009;36:164-9.

5. Fujita Y, Moriyama S, Aoki S, et al. Estimation of the success rate of anesthetic management for thymectomy

Cite this article as: Pompeo E, Elkhouly AG. Spontaneous ventilation thoracoscopic thymectomy: attractive or exceptionable? J Thorac Dis 2018;10(Suppl 33):S3981-S3983. doi: $10.21037 /$ jtd.2018.09.75 in patients with myasthenia gravis treated without muscle relaxants: a retrospective observational cohort study. J Anesth 2015;29:794-7.

6. Al-Abdullatief M, Wahood A, Al-Shirawi N, et al. Awake anaesthesia for major thoracic surgical procedures: an observational study. Eur J Cardiothorac Surg 2007;32:346-50.

7. Pompeo E, Dauri M, Massa R, et al. Minimalist thoracoscopic resection of thymoma associated with myasthenia gravis. J Thorac Cardiovasc Surg 2017;154:1463-5.

8. Tsunezuka Y, Oda M, Matsumoto I, et al. Extended thymectomy in patients with myasthenia gravis with high thoracic epidural anesthesia alone. World J Surg 2004;28:962-5: discussion 965-6.

9. Matsumoto I, Oda M, Watanabe G. Awake endoscopic thymectomy via an infrasternal approach using sternal lifting. Thorac Cardiovasc Surg 2008;56:311-3.

10. Ashour M. Prevalence of ectopic thymic tissue in myasthenia gravis and its clinical significance. J Thorac Cardiovasc Surg 1995;109:632-5.

11. Masaoka A, Yamakawa Y, Niwa H, et al. Extended thymectomy for myasthenia gravis patients: a 20-year review. Ann Thorac Surg 1996;62:853-9.

12. Yim AP. Thoracoscopic thymectomy: which side to approach? Ann Thorac Surg 1997;64:584-5.

13. Mineo TC, Pompeo E, Ambrogi V. Video-assisted thoracoscopic thymectomy: from the right or from the left? J Thorac Cardiovasc Surg 1997;114:516-7.

14. Numanami H, Yano M, Yamaji M, et al. Thoracoscopic Thymectomy Using a Subxiphoid Approach for Anterior Mediastinal Tumors. Ann Thorac Cardiovasc Surg 2018;24:65-72.

15. Mineo TC, Pompeo E, Ambrogi V, et al. Adjuvant pneumomediastinum in thoracoscopic thymectomy for myasthenia gravis. Ann Thorac Surg 1996;62:1210-2.

16. Wang $\mathrm{H}, \mathrm{Gu} Z$, Ding J, et al. Perioperative outcomes and long-term survival in clinically early-stage thymic malignancies: video-assisted thoracoscopic thymectomy versus open approaches. J Thorac Dis 2016;8:673-9. 